INPLASY

PROTOCOL

To cite: Roch et al. The female pelvic floor fascia anatomy: $A$ systematic research and review. Inplasy protocol 202150067. doi:

10.37766/inplasy2021.5.0067

Received: 17 May 2021

Published: 17 May 2021

Corresponding author: Melanie Morin

melanie.m.morin@usherbrooke.ca

Author Affiliation:

Université de Sherbrooke

Support: FRSQ Audace.

Review Stage at time of this submission: Preliminary searches.

Conflicts of interest:

None declared.

\section{The female pelvic floor fascia anatomy: A systematic research and review}

Roch, M1; Gaudreault, N2; Cyr, MP3; Venne, G4; Morin, M55.

Review question / Objective: What is the current state of knowledge regarding the anatomy of the pelvic floor fasciae in human females?

Condition being studied: Anatomy of the pelvic floor fasciae in human females.

Study designs to be included: Observational studies (e.g., case series, cohort, case-control and cross-sectional studies) will be included if they provide an anatomical description of the pelvic floor fasciae. Interventional studies will be included if they describe the anatomy at baseline prior to the intervention. Cadaveric or clinical anatomical studies using dissections, histological and/or biological investigations and/ or imaging will be included. Abstracts, editorials and surveys will be excluded. Systematic reviews will be consulted to retrieve relevant references.

INPLASY registration number: This protocol was registered with the International Platform of Registered Systematic Review and Meta-Analysis Protocols (INPLASY) on 17 May 2021 and was last updated on 17 May 2021 (registration number INPLASY202150067).

\section{INTRODUCTION}

Review question / Objective: What is the current state of knowledge regarding the anatomy of the pelvic floor fasciae in human females?

Rationale: The female pelvic floor is a complex anatomical region that comprises the pelvic organs, muscles, nerves and fasciae. The functional anatomy of the pelvic fascial components is currently misunderstood and underexplored. Despite the increasing scientific interest in fasciae in the last decade, a consensus has not been reached about fascia terminology, definition and classification. Indeed, different terminology can be used to 
describe the same structure or can be used for multiple structures. Therefore, this systematic review's primary objective is to explore and gather the current state of knowledge regarding the fascial anatomy of the pelvic floor in human females.

Condition being studied: Anatomy of the pelvic floor fasciae in human females.

\section{METHODS}

Search strategy: We will search the following electronic bibliographic databases: Scopus (including Embase and MEDLINE) as well as MEDLINE (AMED and CINAHL) on the EBSCOhost research platform. The search strategy will include terms relating to or describing the anatomy of the pelvic floor fasciae (e.g., Gallaudet fascia, perineal membrane and endopelvic fascia). The search will be conducted from inception with no restriction to years. Studies available in French, English, Portuguese, Spanish and Italian will be included. Gray literature will also be searched using conference abstracts from leading societies (e.g., International Continence Society).

Participant or population: Female adult human subjects (cadaveric or living) with or without pelvic floor dysfunctions.

Intervention: None.

\section{Comparator: None.}

Study designs to be included: Observational studies (e.g., case series, cohort, case-control and cross-sectional studies) will be included if they provide an anatomical description of the pelvic floor fasciae. Interventional studies will be included if they describe the anatomy at baseline prior to the intervention. Cadaveric or clinical anatomical studies using dissections, histological and/or biological investigations and/or imaging will be included. Abstracts, editorials and surveys will be excluded. Systematic reviews will be consulted to retrieve relevant references.
Eligibility criteria: Female adult human subjects (cadaveric or living) without pelvic floor dysfunctions. Exclusion: Male, animal.

Information sources: Electronic bibliographic databases: Scopus (including Embase and MEDLINE; contact with authors; grey literature.

Main outcome(s): Qualitative or quantitative data regarding the anatomy of the pelvic floor fasciae located caudally to the parietal pelvic fascia will be investigated. The data pertaining to the origin/insertion, path/course and morphology (e.g., length/ width, area, thickness and number of bands) will be analyzed.

Additional outcome(s): Histological investigation of the anatomy of the pelvic floor fasciae located caudally to the parietal pelvic fascia will be investigated.

Data management: Two review authors will independently screen titles and abstracts of studies retrieved to identify the studies that could meet the inclusion criteria outlined above. Subsequently, the full text of these studies of potential interest will be retrieved and independently assessed by the two review team members. Any selection disagreement between the two review authors will be resolved through discussion with a third author. The extracted information will include: authors; date of publication; study design and setting; study population and participant demographics and baseline characteristics; study methodology; and outcomes (e.g., origin/insertion, path/ course, morphology such as length/width, area, thickness and number of bands and histological assessment). The two review authors will independently extract data; discrepancies will be identified and resolved through discussion with a third author when necessary.

Quality assessment / Risk of bias analysis: The Anatomical Quality Assessment (AQUA) Tool will be used to assess the risk of bias in individual studies. The two review authors who extracted the data will evaluate the risk of bias of each study 
independently. Any disagreement between them over the risk of bias will be resolved through discussion with a third author.

Strategy of data synthesis: The studies included are expected to be heterogeneous in nature owing to the varied methodology and tissues involved, and hence rendering the performance of meta-analysis is inappropriate. Therefore, a narrative analysis will be conducted, and a critical appraisal of what is known will be performed based on the risk of bias assessment. Gaps in knowledge will be identified and recommendations for future research will be formulated. Data will be summarized as described in respective studies using the description of the anatomy. Descriptive statistics, with means and standard deviations for continuous variables, and frequencies and percentages for categorical variables will be synthesized when available.

Subgroup analysis: None.

Sensitivity analysis: None.

Language: Studies available in French, English, Portuguese and Italian will be included.

Country(ies) involved: Canada.

Keywords: Anatomy; Connective tissues; Fascia; Pelvic Floor; Systematic Review; Human Females; Women.

Dissemination plans: A paper will be submitted to a leading journal in this field.

Contributions of each author:

Author 1 - Melanie Roch Conceptualization, methodology, validation, formal analysis, resources, data curation, writing original draft preparation.

Email: melanie.roch@usherbrooke.ca

Author 2 - Nathaly Gaudreault Conceptualization, methodology, validation, writing-review and editing, visualization, supervision, project administration, funding acquisition.

Email: nathalie.gaudreault@usherbrooke.ca
Author 3 - Marie-Pierre Cyr Conceptualization, methodology, validation, formal analysis, resources, data curation, writing review and editing.

Email: marie-pierre.cyr@usherbrooke.ca

Author 4 - Gabriel Venne Conceptualization, methodology, validation, writing-review and editing, visualization, supervision.

Email: gabriel.venne@mcgill.ca

Author 5 - Melanie Morin Conceptualization, methodology, validation, writing-review and editing, visualization, supervision, project administration, funding acquisition.

Email: melanie.m.morin@usherbrooke.ca 